\title{
HIPERINTENSIDADE DE SINAL EM T1 DOS NÚCLEOS DA BASE \\ RELATO DE CASO NA ENCEFALOPATIA PORTAL SISTÊMICA ESQUISTOSSOMÓTICA
}

\author{
MARCOS ALBERTO DA COSTA MACHADO JÚNIOR*, FERNANDO PENA GASPAR SOBRINHO*, \\ VERONNICA ALINE OLIVEIRA BARBOSA*, JOSÉ CARLOS BINA**, HUGO DE SOUSA MATOS*
}

\begin{abstract}
RESUMO - Relatamos o caso de uma paciente portadora de esquistossomose mansônica com encefalopatia portal sistêmica, associada a elevada intensidade de sinal em T1 ao nível dos núcleos da base, devido a deposição de manganês, decorrente da presença de colaterais porto-sistêmicos. Ressaltamos os achados radiológicos por ressonância magnética e fazemos revisão bibliográfica sobre o tema. Desconhecemos relato de tal associação na bibliografia.
\end{abstract}

PALAVRAS-CHAVE: esquistossomose mansônica, encefalopatia portal sistêmica, gânglios da base, manganês, neurorradiologia, ressonância magnética.

\section{Hyperintense basal ganglia on T1 weighted MR images: case report in Manson's Schistosomiasis with portal-systemic encephalopathy}

ABSTRACT - We report a case of mansonic schistosomiasis with portal systemic encephalopathy associated with a high intensity signal in basal ganglia on T1 weighted images due to deposition of manganese in tissues related with portal-systemic collateral vessels. A bibliographic review was done focusing the magnetic resonance findings. To the best of our knowledge, these signal changes have not yet been associated with Manson's schistosomiasis.

KEY WORDS: mansonic schistosomiasis, portal-systemic encephalopathy, basal ganglia, manganese, neuroradiology, magnetic resonance image.

Em 1954, Sherlock utilizou a expressão encefalopatia portal sistêmica (EPS) para denominar uma síndrome neurológica que se manifesta ocasionalmente em pacientes com hepatopatia crônica com shunts porto-sistêmicos ${ }^{1}$. As alterações observadas no exame de ressonância magnética (RM) são caracterizadas por hiperintensidade de sinal em T1 nos núcleos da base, características da EPS ${ }^{2}$. Este achado foi inicialmente descrito por alguns autores em casos associados à insuficiência hepática crônica $^{3-6}$, posteriormente em casos de shunt portal sistêmico sem insuficiência hepática ${ }^{7}$, e, mais recentemente, em pacientes submetidos a nutrição parenteral ${ }^{8,9}$. A sua etiopatogenia está associada com a deposição intracelular de manganês (Mn).

A esquistossomose mansoni é endêmica em regiões da América, África e Oriente Próximo. Está presente em cerca de 53 países. O agente etiológico é o Schistosoma mansoni, um trematoda que tem o homem como hospedeiro definitivo e único reservatório de importância epidemiológica. A doença se caracteriza por expressão clínica variável, desde formas assintomáticas / oligossintomáticas a quadros graves com hipertensão portal e raramente hipertensão pulmonar. A

Estudo realizado no Setor de Neurorradiologia do Hospital São Rafael - Fundação Monte Tabor e no Serviço de Tomografia Computadorizada Salvador, IGASE: *Neurorradiologistas do Serviço de Bioimagem do Hospital São Rafael - Fundação Monte Tabor e do Hospital Salvador IGASE; **Médico Infectologista do Hospital São Rafael e Professor da Disciplina Infectologia da Faculdade de Medicina da Universidade Federal da Bahia. Aceite: 4-dezembro-1998.

Dr. Marcos Alberto da Costa Machado Jr - Praça 2 de Julho 156/1301 - 40080-120 Salvador BA - Brasil. Email:machado@e-net.com.br 
complicação mais importante da esquistossomose mansoni é a hemorragia digestiva secundária a varizes de esôfago. Os ovos do verme e a resposta do hospedeiro à sua presença, constituem os principais elementos patogênicos na esquistossomose crônica ${ }^{10}$.

Nós descrevemos um caso de elevado sinal em T1 ao nível dos gânglios da base numa paciente com EPS portadora de shunt portal devido a hepatopatia esquistossomótica.

\section{RELATO DO CASO}

Paciente do sexo feminino, branca, com 19 anos de idade, proveniente de zona endêmica do interior do Estado da Bahia, com desenvolvimento neuropsicomotor normal, portadora de hepatoesplenomegalia esquistossomótica desde os 6 anos e com diagnóstico definido através de biópsia hepática que mostrava fibrose peri-portal de Symmers e ovos de Schistosoma mansoni nas fezes. Recentemente, desenvolveu desorientação e confusão mental. Ao exame físico, acrescentavam-se os dados de ascite e circulação colateral superficial. Foi submetida a estudo tomográfico computadorizado (TC) de crânio que não evidenciou alterações. A RM mostrou hiperintensidade de sinal em T1 ao nível dos núcleos pálidos, regiões sub-talâmicas e pedúnculos cerebrais. As imagens obtidas em T2 e densidade de prótons não mostraram anormalidades (Fig 1). Havia antecedentes de hematêmese devido a varizes de esôfago diagnosticadas ao exame radiológico contrastado do esôfago-estômago e duodeno.

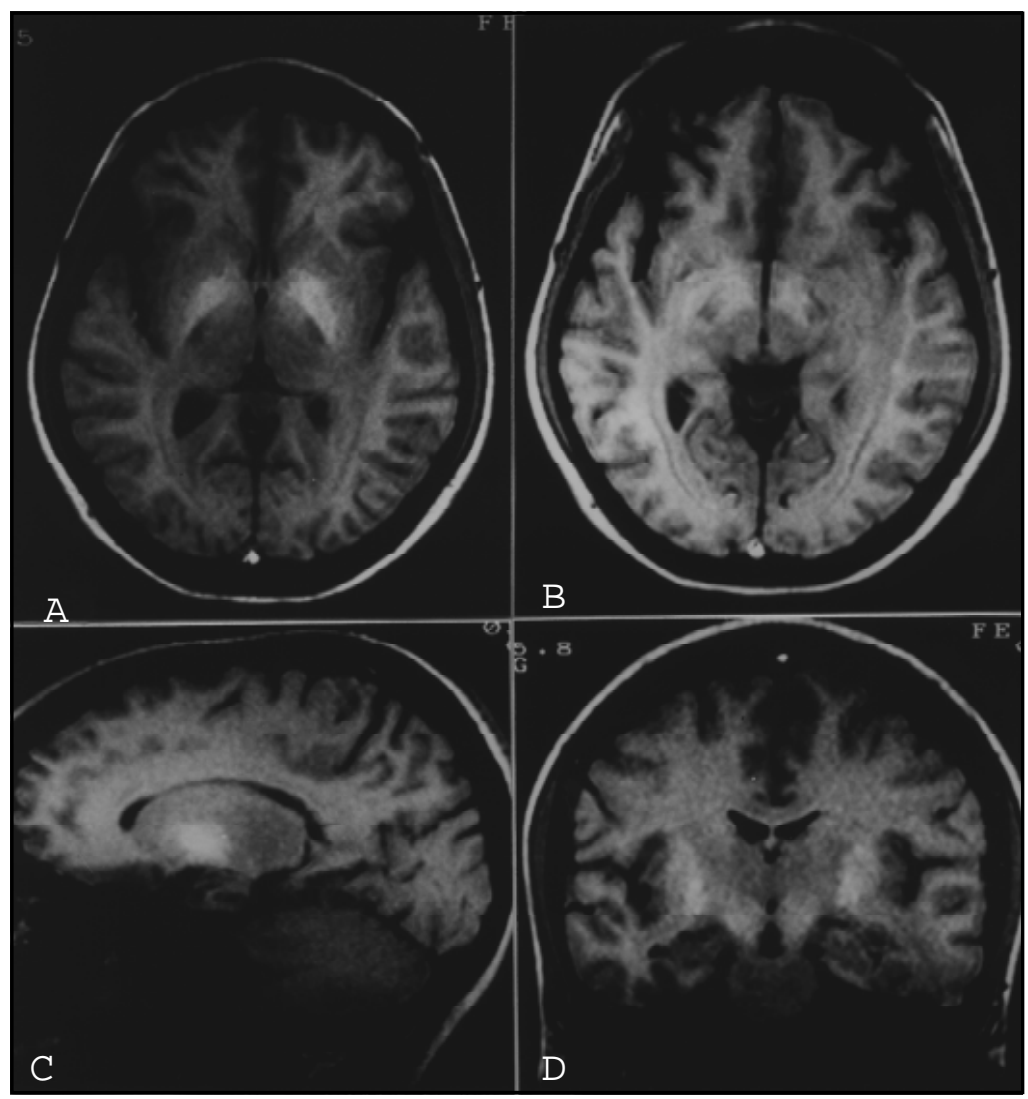

Fig 1. RM (FE-TR 300, TE 14mseg, DEG 90 ). A-B-Axiais, C sagital, D-Coronal. Hiperintensidade de sinal em T1 ao nível dos núcleos pálidos, regiões subtalâmicas $e$ pedúnculos cerebrais. 


\section{DISCUSSÃO}

A EPS ${ }^{1,11}$ é clinicamente diferente da encefalopatia hepática. Esta última ocorre mais comumente em pacientes com cirrose descompensada ${ }^{12}$. Em ambas, as alterações neuropsiquiátricas estão presentes quando coexistem vasos colaterais porto-sistêmicos e podem estar associadas com lesões irreversíveis do parênquima cerebral ${ }^{13}$. Alterações patológicas encefálicas têm sido descritas por diversos autores ${ }^{14-15}$, sendo que a mais importante delas é a atrofia cortical quando associada à cirrose ${ }^{16}$.

Os estudos com TC contribuíram pouco para a compreensão dos mecanismos anatomofisiopatológicos dos distúrbios neuropsiquiátricos. Os achados são inespecíficos, revelando apenas um quadro de atrofia cerebral difusa ${ }^{17-18}$. As primeiras observações das alterações de sinal ao exame de RM ocorreram em 19912,5. O quadro, à RM, é caracterizado por hiperintensidade de sinal em T1 nos núcleos da base (mais comumente), nos núcleos pálidos e, com menor frequência, nos núcleos subtalâmicos, adeno-hipófise e mesencéfalo5.

Inicialmente, foi proposto que este achado seria consequência dos elevados valores de amonemia, resultado da redução na conversão da amônia em uréia por insuficiência hepatocelular ${ }^{19}$. Todavia, a amônia não é substância paramagnética e não determina encurtamento de T1, exceto em elevadas concentrações ${ }^{4,20}$.

A hipótese atualmente mais aceita, que justifique a elevada intensidade de sinal encontrada nos núcleos da base, é a deposição de $\mathrm{Mn}^{21-24}$. O Mn é metal de transição paramagnética com cinco elétrons não pareados com grande momento magnético capaz de promover encurtamento de T1 no exame de RM. Por outro lado, para que se obtenham alterações em imagens em T2 são necessárias concentrações extremamente elevadas de Mn, incompatíveis com a vida ${ }^{25}$.

Newland e col. ${ }^{21}$ relatam hiperintensidade de sinal em T1 ao nível dos núcleos da base e da hipófise após a administração parenteral de $5 \mathrm{mg} / \mathrm{kg}$ de cloridrato de Mn em macacos. Krieger et col. ${ }^{22}$ correlacionam os mesmos achados com níveis de Mn do sangue em pacientes portadores de doença hepática em estágio terminal. Em 1991, Inoue e col. ${ }^{2}$ estudaram a hiperintensidade de sinal dos núcleos da base em pacientes portadores de EPS utilizando a portografia transarterial. Todos os pacientes apresentavam vasos colaterais maiores que $10 \mathrm{~mm}$ de diâmetro, oriundos da veia mesentérica superior. As dimensões dos shunts porto-sistêmicos parecem ser o principal fator anatômico relacionado às alterações dos núcleos pálidos ${ }^{2,26}$

$\mathrm{Na}$ esquistossomose, o verme adulto vive no sistema porta e migra para oviposição na parede intestinal. Muitos ovos desprendem-se para a circulação sistêmica e impactam no fígado e em outros órgãos. A presença dos ovos nos tecidos do hospedeiro promove uma resposta imunológica, traduzida geralmente pelo granuloma esquistossomótico. A lesão hepática básica está em torno do tecido conjuntivo que circunda os ramos intra-hepáticos da veia porta, onde ocorre pileflebite e peripileflebite que levam à perda da elasticidade dos vasos. A injeção de contraste e o molde plástico do sistema porta mostram a rica rede de capilares limitada à zona periportal, porém não servindo de novas vias para o sangue portal. A estrutura lobular permanece conservada. Não há, portanto, cirrose hepática e sim fibrose periportal descrita por Symmers e aceita como patognomônica da esquistossomose. Os ovos de Schistosoma e respectivos granulomas podem ser vistos nos espaços portais e, mais raramente, nos lóbulos. Após a extinção da atividade parasitária, os ovos e os granulomas podem desaparecer completamente, permanecendo, no entanto, as lesões da fibrose de Symmers que podem reverter, total ou parcialmente, com o tratamento específico ${ }^{27,29}$. Na necrópsia de indivíduos com hipertensão porta esquistossomótica não é raro o encontro de trombos no tronco principal da veia porta. A fibrose hepática pode levar à hepatomegalia e, mais tarde, à hipertensão porta com circulação colateral e varizes no esôfago. Na portografia e ecografia, vê-se grande calibre e tortuosidade das veias do sistema porta e circulação colateral ${ }^{10}$.

Em pessoas saudáveis não ocorre deposição de Mn no sistema nervoso central (SNC) devido ao excelente mecanismo de auto-regulação entre o sistema gastrointestinal e o fígado ${ }^{24}$. Normalmente, 
1,0 a 3,5 \% da ingestão oral de Mn é absorvida para a circulação sistêmica ${ }^{30}$, sendo que $98 \%$ dele é metabolizado no fígado e excretado na bile ${ }^{31}$. Contudo, a presença de vasos colaterais porto-sistêmicos pode desviar o Mn do seu metabolismo hepático e levar à sua deposição patológica no $\mathrm{SNC}^{12}$. Uma outra causa de deposição comprovada de Mn, porém sem associação com shunt portal-sistêmico, é o uso de nutrição parenteral por tempo prolongado ${ }^{8,9}$. Maeda e col. ${ }^{6}$, através de autópsia em portadores de EPS, encontraram, no SNC, dosagens de Mn de 4 a 10 vezes maiores que os valores normais.

Toxicidade por Mn tem sido descrita em mineiros expostos à inalação ${ }^{25}$ e em modelos experimentais animais ${ }^{32}$. Os sintomas iniciais decorrentes da deposição de Mn no SNC são inespecíficos, incluindo astenia, anorexia, irritabilidade, insônia, violência incontrolável e labilidade emocional. Langston ${ }^{33}$ refere ainda a ocorrência de fenômenos alucinógenos, alterações da mobilidade ocular e distonias. Progressivamente, podem surgir parkinsonismo, distúrbio da linguagem, bradicinesia, desordens da linguagem e tremores ${ }^{34}$. Em casos crônicos, o principal achado clínico é um distúrbio do movimento similar à doença de Parkinson; provavelmente por depleção dos níveis de dopamina, como resultado da deposição de Mn. Os sintomas geralmente são reversíveis, mas podem deixar sequelas permanentes ou, como descrito recentemente, progredir, a despeito da suspensão da exposição ${ }^{34}$.

A passagem do Mn através da barreira hemato-encefálica (BHE) é mediada pela transferrina. A ação tóxica sobre o tecido nervoso se deve ao Mn 3+ livre e instável com ação oxidante sobre a dopamina e sua transformação em quinona e semiquinona, auto-oxidada em neuromelanina ${ }^{35}$. $\mathrm{O}$ acúmulo de neuromelanina pode ser também responsável pelo encurtamento de T1 ao nível dos núcleos da base ${ }^{7}$.

Existem relatos de desaparecimento das alterações de sinal após transplante hepático ${ }^{3}$, após suspensão da suplementação de $\mathrm{Mn}$ ou da dieta parenteral ${ }^{36-38}$. Recente relato de completo desaparecimento dos achados à RM é feito por Matsumoto e col. ${ }^{12}$, após embolização terapêutica de shunt venoso porto-sistêmico intra-hepático.

Luttmann e col., em 1997, descreveram um caso semelhante de hiperintensidade de sinal em T1 ao nível da substância nigra e tratos corticopontinos, bilateral e simétrica, em paciente com encefalopatia por HIV. Não apresenta explicações para esses achados, mas descarta a possibilidade de causas como o shunt porto-sistêmico e a nutrição parenteral ${ }^{39}$.

Enfatizamos a importância da correta identificação e interpretação das alterações da intensidade de sinal dos núcleos da base, uma vez que a relação de condições associadas àquelas alterações está aumentando progressivamente.

Agradecimentos - Agradecemos ao Técnico de Ressonância Magnética Sr. Lauro Conceição Vieira pela colaboração e ao fotógrafo Tadeu Miranda pelas ilustrações do nosso estudo.

\section{REFERÊNCIAS}

1. Sherlock S, Summerrskill W H, Portal-systemic encephalophaty: neurological complications of liver disease. Lancet 1954;267:453-457.

2. Inoue $\mathrm{E}$, Hori $\mathrm{S}$, Yoshifumi $\mathrm{N}$ et al. Portal-systemic encephalopathy: presence of basal ganglia lesions with high signal intensity on MR images. Radiology 1991;179:551-555.

3. Kulisevsky J, Pujol J, Deus J, et al. Persistence of MRI hyperintensity of globus pallius in cirrotic patients: a 2 year follow up study. Neurology 1995;45:995-997.

4. Kulisevsky J, Pujol J, Junque C,Deus J et al. MRI pallidal hyperintensity and brain atrophy in cirrhotic patients: two different MRI patterns of clinical deterioration? Neurology 1993;43:2570-2573.

5. Brunberg JÁ, Kanal E, Hirsch W et al. Chronic acquired hepatic failure: MR imaging of the brain at 1.5 T. AJNR 1991;12:909-914.

6. Maeda H, Sato M, Yoshikawa WA, et al. Brain MR imaging in patients with hepatic cirrhosis: relationship between high intensity signal in basal ganglia on T1 - weigheted images and elemental concentration in brain. Neuroradiology 1997;39:546-550.

7. Gozzoli L, Ambrogio L, Grasso M, Iperintensita dei nuclei della base nelle sequenze RM T1 -W in un caso di shunt portosistemico senza insufficienza epatica. Riv Neuroradiol 1996;9:493-500.

8. Scott AM, Westrich TJ, Hirsch JD. Hyperintense basal ganglia on T1-weigheted MR images in patients receiving parenteral nutrition. Radiology 1991;181:117-120.

9. Quaghebeur G, Taylor WJ, Kingsley DPE. MRI in children receiving total parenteral nutrition. Neuroradiology 1996;38:680-683. 
10. Prata A, Esquitossomose manosini. In Veronesi R. Doenças infecciosas e parasitárias. 8 Ed. Rio de Janeiro: Guanabara Koogan; 1992:838-855.

11. Summerskill WHJ, Davidson EA, Sherlock S, et al. The neuropsychiatric syndrome associatredd with hepatic cirrhosis and as extensive collateral circulation. QJ Med 1956;25:245-266.

12. Matsumoto S, Mori H, Yoshioka K, et al. Effects of portal-systemic shunt embolization on the basal ganglia: MRI. Neuroradiology 1997;39:326-328.

13. Sherlock S. Disease of the liver and biliary system. 7 Ed. Oxford: Blackwell, 1985.

14. Waggoner RW, Malamud N. Wilson's disease in the light of cerebral changes following ordinary acquired liver disorders. J Nerv Ment Dis 1942;96:410-423.

15. Victor M, Adams RD, Cole M. The acquired (non Wilsonian) type of chronic hepatocerebral degeneration. Medicine 1965;44:345-396.

16. Zeneroli ML, Cioni G, Vezzelli C, et al. Prevalence of brain atrophy in liver cirrhosis patients with chronic persistent encephalopathy: evaluation by computed tomography. J Hepatol 1987;4:283-292.

17. Bernthal P, Hays A. Cerebral CT scan abnormalities in cholestatic and hepatocellular disease and their relationship to neuropsychologic test performance. Hepatology 1987;7:107-114,.

18. Zeneroli ML, Cioni G, Cooper AJL. Prevalence of brain atrophy in liver cirrhosis patients with chronic persistent encephalopathy: evaluation by computed tomograpy. J Hepatol 1987;4:283-292.

19. Kulisevski J, Pujol J, Grau JM. Pallidal hyperintensity on MRI in cirrhotiv patients: clinical correlations. Hepatology 1992;16:1382-1388.

20. Vymazal J, Babis M. T1 and T2 alterations in the brains of patients with hepatic cirrhosis. Am J Neurorad 1996;17:333-336.

21. Newland MC, Ceckler TL, Kordower JH, Weiss B. Visualizing manganese in the primate basal ganglia with MRI. Exp Neurol 1989;106:251-258.

22. Krieger D, Krieger S, Jansen O, Gass P. Manganese and chronic hepatic encephalophaty. Lancet 1955;346:270-274.

23. Mirowitz AS, Westrich TJ, Basal banglia signal intensity alterations: rversal after discontinuation of parenteral manganese adminstration . Radiology 1992;185:535-536.

24. Markesbery WR,, Ehmann WD, Hossain TIM, Alauddin M. Brain manganese concentrations in human aging and Alzheimer's disease. Neurotoxicology 1984;5:49-58.

25. Nelson K, Golnick J, Korn T, Angle C. Manganese encephalopathy: utility of early magnetic resonance imaging. Br J Ind Med 1993;50:510- 513.

26. Pujol A, Pujol J. Hyperintense globus pallidus on T1 - weighetd MRI in cirrhotic patients in associated with severity of the liver failure. Neurology 1993;43:65-69.

27. Andrade ZA. Hepatic Schistosomiasis morphologic aspects. Reprint. In Popper H, Schaffner F (eds). Progress in liver diseases. 1964.

28. Andrade ZA, Bina JC. A patologia da forma hepato-esplênica da esquistossomose mansoni em sua forma avançada. Mem Inst Oswaldo Cruz 1983;78:285-305.

29. Symmers W St C. Note on a new form of liver cirrhosis due to the presence of the ova of bilharzia haematobia. J Path Bact 1904;9:237-239.

30. Greenberb DM, Copp DH, Cuthbertson EM. Studies in mineral metabolism with aid of artificial radioactive isotopes; distribution and excretion particulary by way of bile, of iron, cobalt and manganese. J Biol Chem 1943;147:749-756.

31. Larsen NA, Pakkenberg H, Damsgaard E, Heydorn K. Topographical distribution of arsenic, manganese, and selenium in the normal human brain. J Nerol Sci 1979;42:407-416.

32. Newland MC, Cox C, Hamada R. The clearance of manganese chloride in the primate. Fundam Appl Toxicol 1987;9:314-328.

33. Langston JW. Intustrial toxins in parkinsonism. Annu Course American Academy of Neurology. Vol IV, Course 440. San Fransisco, 1996.

34. Huang CC, Lu CS. Progression after chronic manganese exposure. Neurology1993;43:1479-1483.

35. Donaldson J, La Bella FS, Gesser D. Enhanced autoxidation of dopamine as a possible basis of manganese neurotoxicity. Neurotoxicology 1981;2:53 - 64 .

36. Mirowitz SA, Westrich TJ, Hirsch JD. Basal ganglia signal intensity alterations: reversal after discontinuation of parenteral manganese administration. Radiology 1992;185:535-536.

37. Kulisevsky J,, Pujol J. Persistencee of MRI hyperintnsity of the globus pallidus in cirrhotic patients: a 2 years folllow-up study. Neurology 1995;45:995-997.

38. Devenyi AG, Barron TF. Dystonia, hiperintense basal ganglia, and high wholwe blood manganese level in Alagille's syndrome. Gastroenterology 1994;106:1068-1071.

39. Luttmann S, Husstedt I W, Schuiere. High-signal lesions in the midbrain on T1-weighted MRI in na HIV-infected patient. Neuroradiology 1997;39:136-138. 